\title{
Electron Tomography Reveals Novel Ultrastructure of Mitochondria during Spermatogenesis
}

\author{
G.D. Martens
}

Bioimaging Facility, Department of Botany, University of British Columbia, Vancouver, B.C., Canada, V6T 1 Z4

Spermatogenesis is the differentiation of germ cells into mature sperm and involves the packaging and reduction of cellular contents into an extremely compact, and often motile cell. Novel ultrastructure of mitochondria during spermatogenesis in the marine snail, N. lamellosa is revealed by TEM and electron tomography.

During spermatogenesis, mitochondria are organized in groups of 8 radiating around the axoneme (Fig 1 and 2). As mitochondria organize around the axoneme they reduce in size, become electron dense and morph from traditional tubular shaped to cubical shaped without defined cristae (Fig 1). EM tomography reveals that migrating mitochondria are associated with groups of microtubules outside the fused outer membranes and alongside the axoneme (Fig 2). Figure 1B (white arrow) indicates microtubules possibly linking two adjacent mitochondria. Mitochondria have been shown to migrate along microtubule tracts as well as actin fibers $(1,2)$. Whether or not other cytoskeletal elements play a role in this organization remains to be determined.

EM tomography also shows that as the mitochondria shuttle closer together the outer mitochondrial membranes fuse (Fig. 1 and 2) creating a cylinder of outer mitochondrial membrane. Note that at this stage of differentiation, 7 of 8 mitochondrial outer membranes have fused leaving a gap between two mitochondria (white arrow; Fig 2B) and are sometimes linked via microtubules (white arrow; Fig 1B). Whether this condition persists in the fully differentiated sperm is to be determined. The inner mitochondrial membrane, however, does not fuse (Fig. 1 and 2) creating a structure of highly reduced cubical mitochondria within a tube of outer mitochondrial membrane. This mitochondrial ultrastructure has not been previously reported. This may be a unique situation during spermatogenesis. Mitochondrial fusion has been shown to be a two-stage event with distinct mechanisms $(3,4)$ and disruption of mitochondrial fusion is a hallmark of several diseases in mammals $(4,5)$. I suggest that during spermatogenesis the fusion of inner mitochondrial membrane is inhibited, possibly by down regulation of GTP hydrolysis and/or disruption of the inner membrane potential, allowing for the development of these cylinders of reduced mitochondria.

References

(1) P.J. Hollenbeck and W.M. Saxton, J Cell Science, 118 (2005) 5411

(2) H. Chen and D.C. Chan, Curr. Top. Dev. Biol., 59 (2004) 119

(3) S.L. Meeusen and J. Nunnari, Science, 305 (2004) 1747

(4) S.L. Meeusen and J. Nunnari, Curr. Opinion Cell Biol. 17 (2005) 389

(5) S. Zuchner, et al., Nat. Genet. 36 (2004) 449 

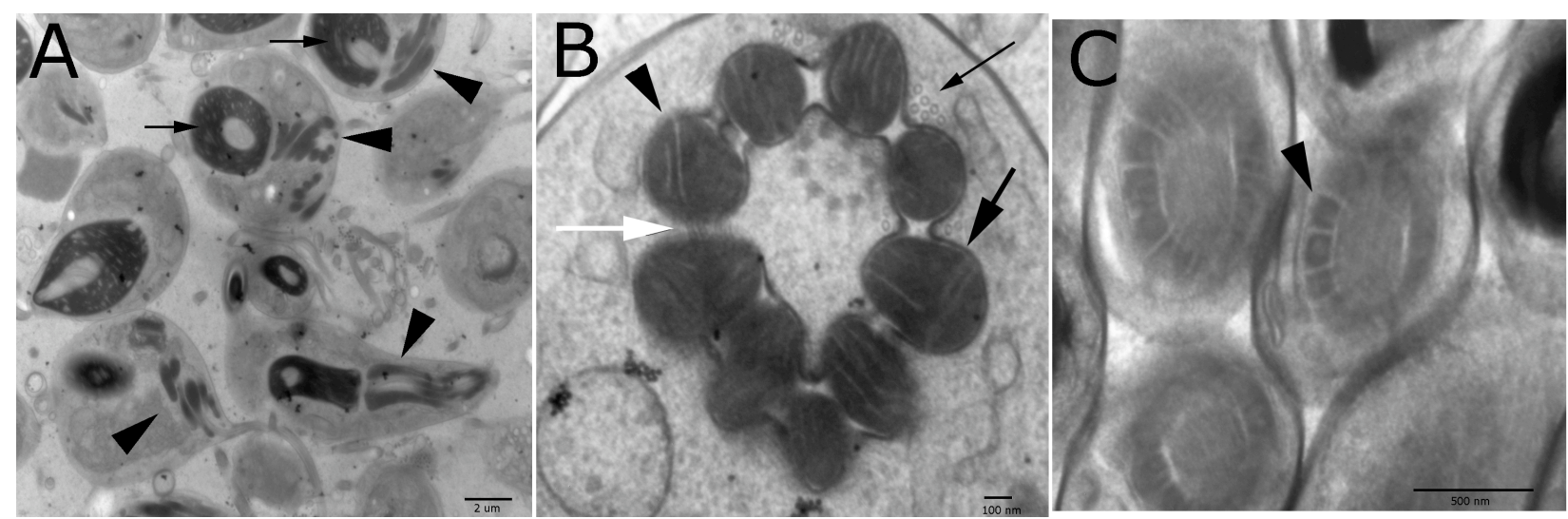

Figure 1. TEM micrographs of high-pressure frozen testis of N. lamellosa. A. Arrows indicate the nucleus of developing sperm. Arrowheads indicate differentiating mitochondria. Scale $2 \mathrm{um}$. B. Mitochondria (black arrowheads)are typically arranged into a bundle of 8 (however, this example indicates 9 mitochondria) radiating around the axoneme. Large black arrow indicates fused outer membrane between adjacent mitochondria. Small black arrow points to a group of microtubules. White arrow points to a gap between microtubules where the outer membrane has not fused. There are also microtubules present, possibly linking the two mitochondria. Scale $100 \mathrm{~nm}$. C. Fully differentiated mitochondria (black arrow head) showing cubic shape. Scale $500 \mathrm{~nm}$.

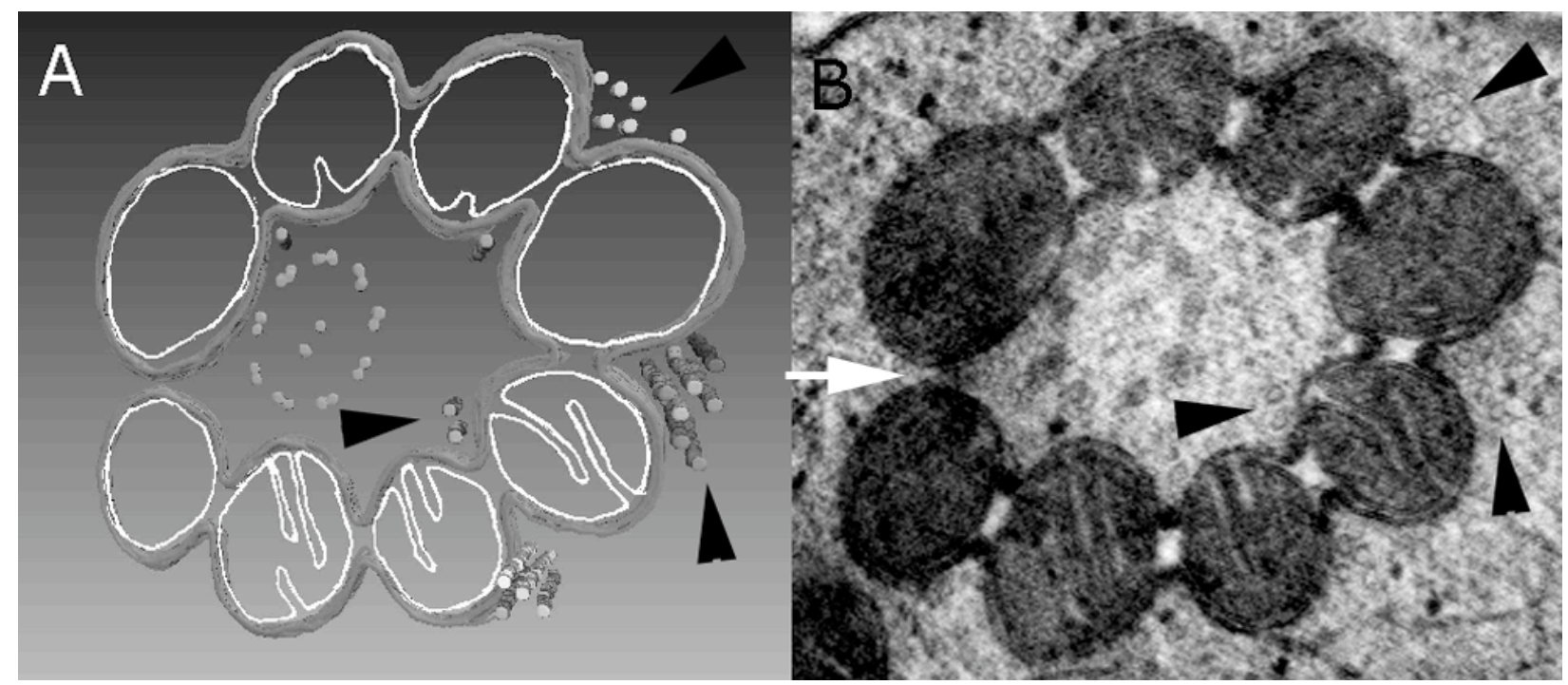

Figure 2. A. Partial tomography model of mitochondria and the fusing of the outer membranes during spermatogenesis of $N$. lamellosa. B. Single image from which the partial model in A was created. Arrowheads point to the same microtubule groups in both A and B. The white arrow in $B$ points to a gap between adjacent mitochondria where the outer membranes have not fused. 